\title{
SOFTWARE PARA FACILITAR LA CORRECTA IMPLEMENTACIÓN DEL MÉTODO NATURAL EN EL PARKOUR
}

Jefferson Velasco Beltrán, Gabriel Pérez Moreno y Diego Darío López Mera

Semillero de Investigación ITmedia

Institución Universitaria Antonio José Camacho

Recepción: 31/07/2019. Aceptado: 30/10/2019.

Cómo citar este artículo:

Velasco Beltrán, J., Pérez Moreno, G. \& López Mera, D.D. (2019). Software para facilitar la correcta implementación del método natural en el parkour. Revista Sapientía, 11 (22), 52-59.

\section{RESUMEN}

El parkour es una disciplina deportiva que gana cada vez más adeptos en el mundo; no obstante, en muchos casos, estos practicantes inician de forma empírica y sin la supervisión de un profesional del deporte. Este artículo describe el desarrollo de un aplicativo para dispositivos móviles que sirva como herramienta que apoye al practicante a desarrollar un marco de buenas prácticas en los entrenamientos. Para esto, el aplicativo describe una serie de rutinas de ejercicios, fundamentadas en el Método Natural de Georges Hébert.

\section{PALABRAS CLAVE:}

Dispositivo Móvil, Georges Hébert, Método Natural, Parkour, Rutinas.

\section{ABSTRACT}

Parkour is a sports discipline that is gaining more and more followers in the world; however, in many cases, these practitioners start empirically and without the supervision of a sports professional. This paper describes the development of an application for mobile devices that serves as a tool that supports the practitioner to develop a framework of good practice in training. For this, the application describes a series of exercise routines, based on the Georges Hébert's Natural Method

\section{KEYWORDS:}

Georges Hébert, Mobile Device, Natural Method, Parkour, Routines.

\section{INTRODUCCIÓN}

El parkour es una disciplina deportiva de origen francés que basa sus fundamentos en el modelo de gimnasia denominado Método Natural. El parkour también es conocido como "el arte del desplazamiento", y consiste en desplazarse en cualquier entorno, usando las habilidades del propio cuerpo, 
procurando ser lo más rápido y fluido posible, y efectuando movimientos seguros y eficientes. Esto implica superar obstáculos que se presenten en el recorrido (Scarnatto y Díaz, 2010; Martínez, 2015). A los practicantes del parkour se les conoce como traceurs o traceuses, en el caso de las mujeres.

En Latinoamérica, este deporte está ganando cada vez más seguidores. En Cali, por ejemplo, la Secretaría del Deporte y la Recreación cuenta con el programa Vértigo que fomenta la práctica de deportes extremos, entre los que se incluye el parkour (El País, 2018).

Sin embargo, la práctica sin guía o poco controlada del parkour no está exenta de golpes, heridas o fracturas óseas, además de lesiones internas graves (Vivanco et al, 2013). Inclusive un inadecuado calentamiento antes de empezar la actividad puede causar otras lesiones como tendinitis rotulianas, epicondilitis y esguinces de tobillo y muñeca (Lledó, 2017). Así que el uso adecuado del Método Natural puede prevenir las lesiones por la práctica del parkour, al menos las que no estén relacionadas con accidentes por la naturaleza extrema de este deporte.

Como una propuesta para mejorar la práctica del parkour, se diseñó y desarrolló una aplicación para teléfonos móviles, con el fin de que los traceurs puedan realizar rutinas de ejercicios basadas en el Método Natural, y llevar un registro y control de sus prácticas.

En el mercado se encuentran aplicaciones como herramientas para la guía y fomento del entrenamiento físico en general, sin basarse en el Método Natural (Runkeeper de ASICS Digital, NRC de Nike o Zombies Run! de Six to Start y Naomi Alderman). Inclusive existen dos aplicaciones relacionadas con la práctica del Parkour: Parkour Training de Free Apps Laborator y Ultimate Parkour de Nicolas Provost. Sin embargo, ambas aplicaciones sólo muestran vídeos de traceurs en sus prácticas, pero no explican un paso a paso de las rutinas; aparte de que el contenido se encuentra exclusivamente en inglés.

En el desarrollo de este proyecto se usó el framework Ionic para la creación de aplicaciones móviles apoyándose en las tecnologías web (Griffith, 2017; Madera, 2018); el paquete de herramientas de Google Cloud (Firebase, autenticación de usuarios, notificaciones push) (Smyth, 2017); el cliente del framework Angular (Rivero, 2016); las hojas de estilo de Sass CSS; el framework de desarrollo móvil para el manejo de las API's nativas de un dispositivo móvil Apache Cordova (Wargo, 2015), y el manejador de paquetes de instalación NPM.

\section{EL MÉTODO NATURAL EN EL PARKOUR}

El Método Natural es un sistema de gimnasia creado en el siglo XX, el cual tiene sus orígenes en los postulados formulados por el oficial de la marina francesa Georges Hébert (1875-1957). Debido a sus viajes a las colonias francesas, al oficial le llamó la atención la sobresaliente condición física de las tribus de esas zonas. Hébert identificó que estos habitantes no consideraban sus actividades como ejercicios de fortalecimiento físico, sino como algo propio de su cotidianidad (Santandreu, 2013).

En general, el Método Natural antepone el desarrollo integral del físico al de la necesidad de ejercitarse con fines competitivos; puesto que expresa que el cuerpo debe ser la herramienta necesaria para darle valor al desarrollo de la mente, y que el altruismo es la conducta que debe regir la vida del ser humano (Gálvez, 2011).

El Método Natural se divide en diez familias fundamentales de ejercicios, las cuales buscan utilizar todas las partes del cuerpo: marcha, carrera, salto, cuadrupedia, trepar, equilibrismo, lanzamiento, levantamiento, defensa y natación (Soares, 2003). Estos ejercicios se realizan tanto en plateaus, es decir, lugares especialmente acondicionados, como en parcours, y recorridos en la naturaleza (Sampayo, 2013). 
De estas familias, la defensa hace referencia a la habilidad propia del ser humano de defenderse mediante diferentes técnicas de derribamiento corporal. El levantamiento y lanzamiento, por su parte, aplican a las actividades donde se necesita mover objetos, ya sea para un trabajo específico o para defenderse en ciertas circunstancias, procurando siempre evitar al máximo la confrontación. Por otro lado, la cuadrupedia procura el uso de las cuatro extremidades corporales; por ejemplo, se habla de cuatro apoyos cuando se hacen movimientos en el suelo similar a los animales cuadrúpedos.

Ahora bien, en cuanto al parkour, a mediados de los años noventa, Raymond Belle, un ex-militar y bombero de profesión, buscaba la forma de entrenar a jóvenes del suburbio parisino de Lysses, buscando ocupar sus tiempos libres en algo más constructivo que el ocio. Es allí que, inspirado en el Método Natural, decide inculcar sus principios en aquellos jóvenes. De esta forma, junto con su hijo, David Belle, y Sebastien Foucan desarrollan la disciplina física conocida como parkour. En esta se busca que, a través de la apropiación de los espacios urbanos, se pueda lograr un alto grado de desempeño físico. De todo esto se llega a la idea que termina siendo el eje central del parkour: llegar del punto A al B de la forma más fluida y eficiente posible, dejando de lado todos los movimientos que busquen la exaltación de lo estético y que vayan en contra de lo funcional. De tal modo que el parkour es una disciplina que utiliza el Método Natural, y en la que lo primordial es el desarrollo personal y no tanto la competición (Leyden, 2013).

\section{METODOLOGÍA}

Esta fue una investigación aplicada, en la que a través de la tecnología se quiso ofrecer una herramienta para mejorar la práctica del parkour. Inicialmente, la investigación partió de observar cómo algunos practicantes del parkour o traceurs no le prestan mucho cuidado al marco de desarrollo implementado en el Método Natural para optar por un aprendizaje empírico que, en la mayoría de los casos, repercute negativamente en el estado físico.

De tal modo que el proyecto abarcó las fases de análisis, diseño y desarrollo.

En la primera fase se entrevistaron a traceurs de la ciudad de Cali (colectivo 'Parkour para la gente'), con el propósito de registrar sus impresiones de lo que debería incluir un software destinado al parkour.

También se evaluaron las tecnologías a utilizar en el desarrollo de la aplicación.

La fase de diseño inició con la elaboraron de las historias de usuario con el propósito de tener una base con la cual identificar la información y contenidos principales de la aplicación. Se modeló la base de datos al igual que la arquitectura del sistema.

En la fase de desarrollo se utilizaron tecnologías como Ionic y Firebase para la creación del aplicativo.

Para esta metodología se usó el marco Scrum para agilizar el desarrollo.

\section{RESULTADOS}

Se diseñó y desarrolló una aplicación móvil para ayudar a los traceurs, principalmente en llevar el control y gestión de las actividades propias del parkour.

En un principio se tuvo planeado desarrollar una aplicación nativa para teléfonos móviles, especialmente orientada a Android, ya que este es el sistema operativo más usado en Colombia. Según la revista Portafolio, en el 2018 más del 50\% del mercado colombiano en telefonía móvil fue acaparado por marcas como Samsung, Huawei y Alcatel. Todas con el sistema operativo Android. Frente a 
un $2 \%$ de ventas del iPhone. Sin embargo, a nivel global, la venta de iPhones ocupa un destacado segundo puesto (Chiquiza, 2019). Así que se optó por usar una herramienta que permite el desarrollo de aplicaciones híbridas. La elección fue Ionic, la cual tiene su mayor fortaleza en la premisa de usar el conocimiento en desarrollo web para crear las aplicaciones móviles.

De esta forma, la aplicación móvil como resultado de esta investigación es híbrida y permite lo siguiente:

- Control y registro de los entrenamientos de cada practicante.

- Control de los tiempos invertidos en la ejecución de cada rutina de ejercicios, las cuales fueron divididas en básicas, medias y avanzadas. Cada rutina está compuesta por varios ejercicios. En la Figura 1 se muestra la pantalla de la aplicación en la que se listan algunas de estas rutinas, mientras que la Figura 2 se presenta la descripción de uno de los ejercicios propuestos al traceur. Una vez se seleccione la rutina, la Figura 3 muestra la pantalla en la que se cronometra el tiempo que tarda un traceur en realizar cada uno de los ejercicios de la rutina.

- Indicación de los diferentes músculos involucrados en el desarrollo de cada rutina. La Figura 4, por ejemplo, muestra la pantalla en la que indica los músculos que se fortalecerán durante la ejecución de la rutina.

- Visualización a través de gráficos estadísticos de los avances o retrocesos alcanzados por la realización de las rutinas de cada practicante, con los datos recolectados durante la ejecución de la misma (ver Figura 5).

- Registro de nuevos lugares de entrenamiento, también denominados spots. La Figura 6 muestra la pantalla de registro de un spot, el cual puede ser comentado o calificado por otros usuarios del aplicativo.

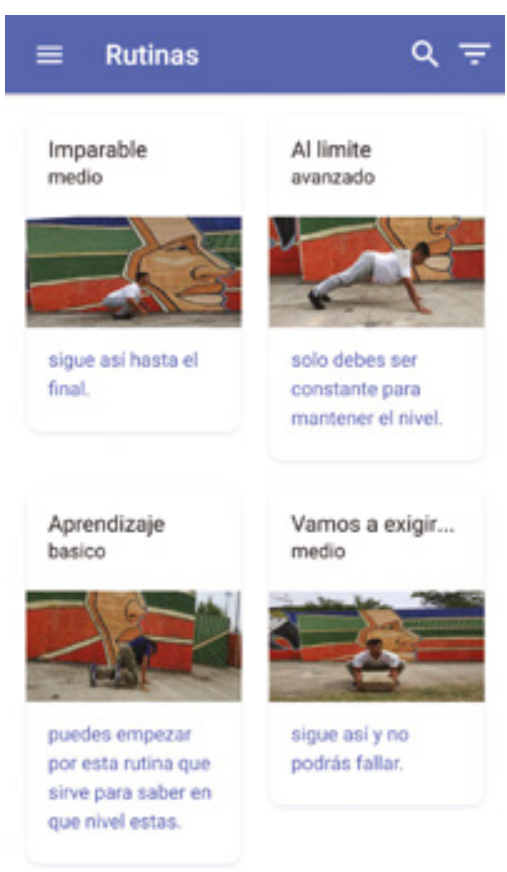

Figura 1. Pantalla de rutinas de entrenamiento. Fuente: elaboración propia.

abdominales $\quad \times$
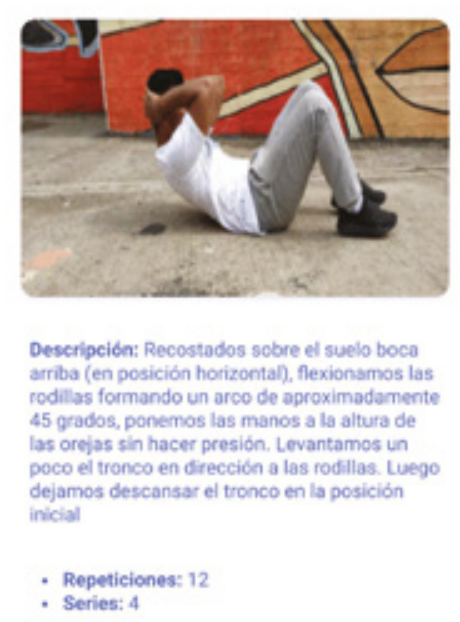

Figura 2. Pantalla en la que se describe el ejercicio de las abdominales. Fuente: elaboración propia. 


\section{$\equiv$ Rutina}

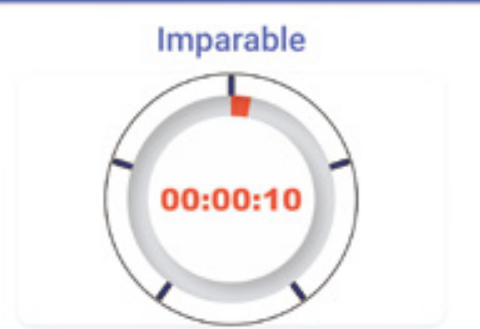

Ejercicio 1 de 5

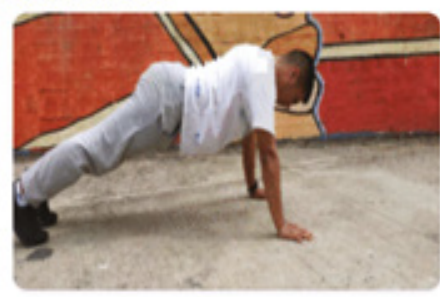

Series: 3 | Repeticiones: 10

\section{CANCELAR}

\section{SIGUIENTE}

Figura 3. Pantalla en la que se cronometra la realización de los ejercicios por rutina. Fuente: elaboración propia.

\section{$\leftarrow \quad$ Imparable (medio)}

Sique asi hasta el final. A continuación listaremos los músculos a fortalecer durante el desarrollo de la rutinax
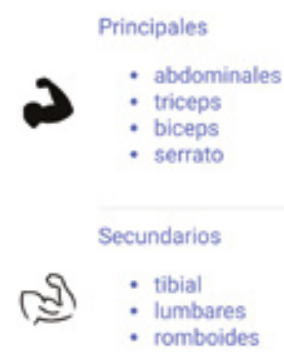

La rutina está compuesta por 5 ejercicios

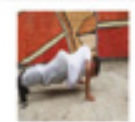

plancha abajo

Repeticiones: 10

Series: $\times 3$

\section{$\downarrow$}

\section{Comenzar}

Figura 4. Pantalla de rutina de nivel medio, compuesta por cinco ejercicios. Fuente: elaboración propia.
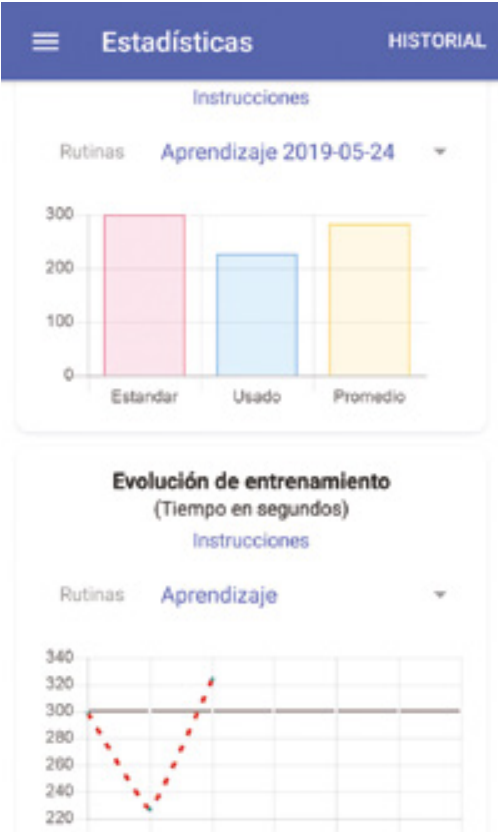

Figura 5. Pantalla de gráficos estadísticos. Fuente: elaboración propia.
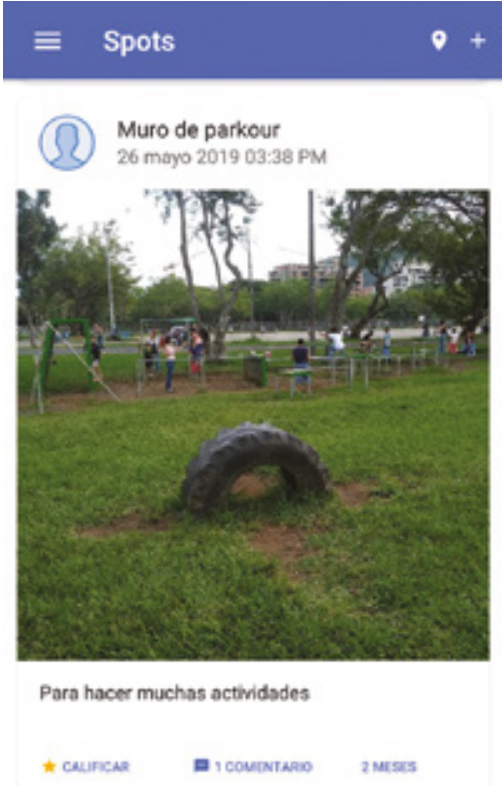

Figura 6. Pantalla registro de spots. Fuente: elaboración propia. 


\section{CONCLUSIONES}

El parkour es un deporte en el que se trabaja muscularmente todo el cuerpo; sin embargo, por su naturaleza de deporte extremo, puede ser de alto riesgo para sus practicantes. La situación es que la cantidad de seguidores del parkour va en aumento, sobre todo jóvenes que lo practican de forma amateur, emulando acrobacias que ven en vídeos y sin tomar en cuenta el Método Natural. En este deporte, las lesiones no sólo son a causa de golpes o caídas; sino, además, por la repetición de incorrectos movimientos corporales que con el tiempo causan graves traumatismos.

Ahora bien, el Método Natural no es la solución para evitar cualquier tipo de lesión en el parkour; pero, ayuda a prevenir. En este sentido, la aplicación desarrollada es una herramienta que sirve de guía de ejercicios para los practicantes de parkour.

Así que en el aplicativo se tuvo un especial cuidado en el diseño de la interfaz gráfica, pues, esta es el primer punto de contacto e interacción con los usuarios. Para ello, se realizaron diagramas y mockups para determinar el diseño, y se modificaron progresivamente en el tiempo hasta dar con un diseño idóneo. De este modo, cada una de las rutinas de ejercicios son explicadas paso a paso de forma textual y a través de videos cortos, con el propósito de ayudar a mejorar las prácticas y, desde luego, prevenir lesiones.

De todas formas, es importante aclarar que soluciones tecnológicas como la desarrollada deben ser utilizadas con prudencia y responsabilidad, ya que no reemplazan al asesoramiento personalizado de un entrenador experto en parkour.

\section{RECOMENDACIONES Y TRABAJO FUTURO}

La aplicación desarrollada cumple con los objetivos para los cuales se destinó. Sin embargo, es una primera versión de un software y como tal puede mejorarse a medida que más usuarios la utilicen y estén dispuestos a ofrecer retroalimentación. Para esta primera versión se fijó una cierta cantidad de rutinas, así que para un trabajo futuro se podría construir una estrategia para que los usuarios diseñen y compartan nuevas rutinas.

Otra posibilidad es la de incluir una funcionalidad que permita generar recomendaciones de nutrición ideal, con base en las necesidades puntuales de cada tipo de rutina.

También se podría añadir nuevos gráficos estadísticos de consulta que le permitan al usuario interpretar fácilmente los datos generados a través de cada entrenamiento. Por otra parte, esta aplicación podría incluir más funcionalidades de redes sociales online (foros, chat, por ejemplo), con el propósito de facilitar la comunicación.

Por último, la aplicación podría diseñarse de modo tal que pueda ser utilizada por otros dispositivos como los relojes inteligentes, ya que esto permitiría mayor libertad a la hora de realizar actividades físicas.

\section{REFERENCIAS BIBLIOGRÁFICAS}

El País. (mayo 18, 2018). La Secretaría de Recreación de Cali apuesta por el deporte extremo. El País. Recuperado de https://www.elpais.com.co/deportes/la-secretaria-de-recreacion-de-cali-apuesta-por-el-deporte-extremo.html

Gálvez, E. (2011). El Método Natural de Georges Hébert: una aproximación a la educación física en el Real Decreto de Educación Española. Lecturas: Educación física y deportes, (160), 5-13. Recuperado de https://dialnet.unirioja.es/descarga/articulo/4503583. pdf

Griffith, C. (2017). Mobile App Development with Ionic, Revised Edition: Cross-Platform Apps with Ionic, Angular and Cordova. O’Reilly Media. 
Chiquiza, J. (enero, 2019). Estas son las marcas de teléfonos móviles que más se venden en el mundo. Diario La República. Recuperado de https://www. larepublica.co/internet-economy/samsung-appley-huawei-las-marcas-de-celulares-mas-vendidas- 2822540

Leyden, J. (2013). Parkour, cuerpos que trazan heterotopías urbanas. Revista Colombiana de Antropología, 49 (2), 41-61. Recuperado de http://www.redalyc. org/pdf/1050/105029983003.pdf

Lledó, F. (noviembre 27, 2017). Parkour y las lesiones. Fisioterapia Lledó. [Blog]. Recuperado de https:// www.fisiolledo.es/parkour-las-lesiones

Madera, L. (2018). Estudio del framework Ionic 2 para el desarrollo de aplicaciones móviles híbridas (Trabajo de grado). Ingeniería en Sistemas Computacionales. Facultad de Ingeniería en Ciencias Aplicadas. Universidad Técnica del Norte. Ecuador.

Martínez, C. (2015). Lo sublime de la acción: riesgo, límite y frontera a través del parkour (Doctoral dissertation, Universidad de Salamanca). Recuperado de https://dialnet.unirioja.es/servlet/tesis?codigo $=155591$

Portafolio. (octubre 5, 2018). Así se reparte la 'torta' en el mercado de celulares en Colombia. Revista Portafolio. Recuperado de https://www.portafolio.co/ negocios/empresas/asi-se-reparte-la-torta-en-elmercado-de-celulares-en-colombia-521967

Rivero, K. (2016). Angular JS. British Columbia, Leanpub.

Sampayo, D. (2013). Método natural. UMparkour. Recuperado de http://www.umparkour.com/natural. html

Santandreu, F. (diciembre de 2013). Prehistoria del parkour. Parkour Uruguay. Obtenido de http://www. parkour.com.uy/articulos.html
Scarnatto, M. y Díaz, J. (2010). De peatón a traceur en una diagonal. El Parkour en la ciudad de La Plata. Nuevas prácticas, patrimonios motrices y formas de socialidad. In IV Jornadas de Investigación en Educación Corporal 7-9 de octubre de 2010 La Plata, Argentina. Universidad Nacional de La Plata. Facultad de Humanidades y Ciencias de la Educación. Departamento de Educación Física.

Smyth, N. (2017). Firebase Essentials-Android Edition. Payload Media.

Soares, C. (2015). Umaeducacão pela natureza: o método de educacão física de Georges Hébert. Revista Brasileira de Ciências do Esporte, 37 (2), 151-157.

Vivanco, A. et al. (2013). Parkour: una nueva causa de lesiones internas graves. Anales de Pediatría, 79(6), 396-397. Recuperado de http://dx.doi.org/10.1016/j. anpedi.2013.03.004

Wargo, J. (2015). Apache Cordova 4 Programming. Indiana, Pearson Education. 


\section{AUTORES}

Jefferson Velasco Beltrán: Practicante de parkour. Estudiante de ingeniería de sistemas de la Institución Universitaria Antonio José Camacho y Tecnólogo en sistemas de información de la misma institución, graduado con el trabajo de grado "Sistema para el control nutricional a través del código de barras de los productos alimenticios". Correo electrónico: jeffersonvelasco@outlook.com

Gabriel Pérez Moreno: Practicante de parkour. Desarrollador de software en las empresas VOV Solutions y Grupo AFL. Actualmente trabaja en esta última. Es estudiante de ingeniería de sistemas de la Institución Universitaria Antonio José Camacho. Correo electrónico: gabriel06241@gmail.com

Diego Darío López Mera: Profesor de la Institución Universitaria Antonio José Camacho y tutor del semillero de investigación Itmedia. Actualmente lidera un proyecto de investigación relacionado con el diseño de estrategias didácticas basadas en juegos y narrativas transmedia. Es magíster en Diseño y creación interactiva de la Universidad de Caldas e Ingeniero de sistemas de la Universidad del Valle. Correo electrónico: dlopez@admon.uniajc.edu.co 